\title{
Inpatient Perception on Hospitalization Experience across US 50 States: Analysis of the Historical Series from 2007 To 2014
}

\section{*Corresponding Author} \\ Chen-Ching Tang \\ Article History \\ Received: 16.01 .2020 \\ Accepted: 23.01 .2020 \\ Published: 10.02 .2020
}

Szu-Hau Chen ${ }^{1}$, Tsair-Wei Chien ${ }^{2}$, Chen-Ching Tang ${ }^{1 *}$

${ }^{\mathrm{T}}$ Department of Family Medicine, Chi-Mei Medical center, Tainan, Taiwan

${ }^{2}$ Research Departments, Chi-Mei Medical Center, Taiwan

\begin{abstract}
Background: There are many ways to gather and assess patient expectations, experience, and satisfaction, but few use visual representations to report their results on the Google maps and help patients select the best hospitals for consultation. Objectives: To analyze 10-dimensional scores of states in the USA about inpatients' perceptions of their hospitalization experience to develop a method for analyzing data on the Google maps and getting feedback on the smartphones. Methods: We downloaded HCAHPS (Hospital Consumer Assessment of Healthcare Providers and Services) data from the 2007 to 2014 summary of survey results to study (1) whether the 10 dimensional scores can be combined to determine its unidimensionality using Rasch continuous item responses, (2) what type of trends about inpatient perception on hospitalization experience that can be reported with an individual and an overall base, (3) what an online dashboard that can be designed using the Google maps for comparing results of each US state, and (4) how to demonstrate an online assessment that uses smartphones for gathering perceptions of their hospitalization experience in the future. Results: The ten core dimensional scores of each US state about inpatient hospitalization experience reported by the HCAHPS can be unidimensional. The improvement was evident of inpatient perception on hospitalization experience in the historical series. Online visual representation of the Google maps can be easy to build and allows for real-time identification in comparison with the performance of each state. A smartphone app was designed to get feedback directly from patients. Conclusions: We verified that the 10-dimensional scores reporting patient satisfaction in US states could be a unidimensional scale and use Rasch continuous item responses to show results on the Google maps.
\end{abstract}

Keywords: Smart phone, unidimensionality, HCAHPS, hospitalized experience perception, the Google maps SUDPAR.

\section{INTRODUCTION}

The quality of healthcare is an essential topic in the healthcare settings [1]. There are many ways to report the healthcare quality to the public. However, those professional indicators are so unfamiliar to patients, and thus pretty hard to give patients a distinct idea of where to get the best care [2-6].

The England Picker Institute Europe (EPIE) [7] and the Hospital Consumer Assessment of Healthcare Providers and Services (HCAHPS) [8] are two examples of periodically assessing patient expectations of hospital healthcare and of publicly reporting their perceptions of their experience [9-11]. From which, Picker's item-by-item box plots of disclosure and the HCAHPS hospital characteristics comparison charts are the additions to the summary analyses on their respective websites. Whether those items can form a single construct for a more focused insight and examination is required to explore [11].

Psychometrically, we are interested in identifying aberrant survey respondents and selecting the best and worst respondents [12]. A method for distinguishing the best and worst clusters is required using the multiple variables of the assessed dimensional scores for an investigation.

Copyright @ 2020: This is an open-access article distributed under the terms of the Creative Commons Attribution license which permits unrestricted use, distribution, and reproduction in any medium for non commercial use (NonCommercial, or CC-BY-NC) provided the original author and source are credited. 
The HCAHPS inpatient experience questionnaire can be used in four survey modes [13]: postal mail, telephone, postal mail with telephone follow-up, or active interactive voice recognition (IVR), each of which requires efforts to collect data from patients. As smartphones become ubiquitous in the healthcare setting [14], it is necessary to show whether it is possible to offer an alternative survey mode of online assessment using them for a fifth survey mode.

The aim of the current study was thus to investigate (1) whether the inpatient experience item scores can be combined, (2) what statistical method can objectively identify the best and the worst clusters so that they can be used to report the performance of a hospital (or US states in this study), and (3) how to demonstrate an online assessment that uses smartphones for gathering patients' perceptions of their hospitalization experience.

\section{METHODS Study Data}

We downloaded data (Summary Analysis of HCAHPS survey results: July to June from 2007 to 2014 Discharges) of inpatient perceptions of their hospitalization experience across 50 US states (excludes Washington, DC, and the territory of the United States Virgin Islands) at the HCAHPS website [8]. The freely available spreadsheet includes 10 dimension scores (range: 0-100 [higher is better]) consisting of (1)Communication with Nurses, (2)Communication with Doctors, (3)Responsiveness of Hospital Staff, (4)Pain Management, (5)Communication About Medicines, (6)Cleanliness of Hospital Environment, (7)Quietness of Hospital Environment, Information, (9) Overall Hospital Rating, and (10) Recommend the Hospital.

(8)Discharge

\section{Examination of Item Scores Addible}

A Microsoft Excel module was programmed to deal with Rasch [15] continuous item responses (CIR for short) [16], see Multimedia Additional file 1, for examining scale unidimensionality with the criterion of item Infit mean square error (Infit MNSQ) less than 1.5 [17]. The Wright Map (or named person-item map) [18, 19] were drawn using the CIR module.

\section{Picker's item-by-item box plots}

The Picker-type item-by-item box plots similar to the KIDMAP [20, 21] were designed by using Excel VBA (Visual Basic for Applications) program language, see Additional file 1. The Outfit MNSQs for persons less than 2.0 indicates person responses without particularly aberrant or careless behaviors [22]. The squared standardized residuals ( $Z_{n j}=\sqrt{R_{n j}^{2} / \operatorname{Var}_{n j}}$ expected value [22].

\section{Designing a dashboard for comparing results of each US state}

An online dashboard was designed using the google maps [23] for comparing inpatient perceptions on hospitalization of each US state. An additional file 2 is available for the details.

\section{An online assessment using smartphones}

An online app was designed for patients to report their perceptions of their inpatient hospitalization experience. The HCAHPS 25-item questionnaire (see Additional file 3) was used in the app. Four questions were designed to automatically select different paths after they have been answered. For example, if a patient responds "No" to question 10 (During this hospital stay, did you need help from nurses or other hospital staff to get to the bathroom or to use a bedpan?), the app will skip questioning 12, but it will go on to question 11 if the patient answers "Yes". This means that the app will consecutively check each question's response and respond accordingly, which will make the app more convenient and practical to use.

\section{Statistical Analysis}

In the author-made CIR module, $\mathrm{R}^{2}$ was calculated using the regression equation (= $\left.\sum_{n=1}^{N} \sum_{j=1}^{L}\left(E_{n j}-\overline{X_{i j}}\right)^{2} / \sum_{n=1}^{N} \sum_{j=1}^{L}\left(X_{n j}-\overline{X_{i j}}\right)^{2}\right)$. The $t$-value for the item difficulty difference between two items (e.g., infill and dwell volumes) was computed using a comparison equation for two independent variables (= $\left.\left(\delta_{a}-\delta_{b}\right) / \sqrt{1 / \operatorname{Var}_{a}+1 / \operatorname{Var}_{b}}\right)$. The Paired- $t$ and independent- $t$-tests for two variables were used to compare the raw scores, percentage scores, and Rasch expected response scores using any 2 -variable dataset of domains. The person 
OUTFIT MNSQ was obtained using the two values of standardized residual (Z) and item length (L) in the equation (= $\left.\sum_{j=1}^{L} Z_{n j}^{2} / L\right)$

\section{RESULTS}

Trends in Each Domain across Years

Trends of each domain across years were shown in Figure-1. All domain scores are increasing from 2007 to 2014. The easier domains with higher scores are Discharge Information, Communication with Doctors, and Communication with Nurses. The most difficult domains with lower scores are Overall hospital rating, Quietness of Hospital Environment, and Communication About Medicines. Interested readers are suggested to see the results on the Google maps at the reference [24].
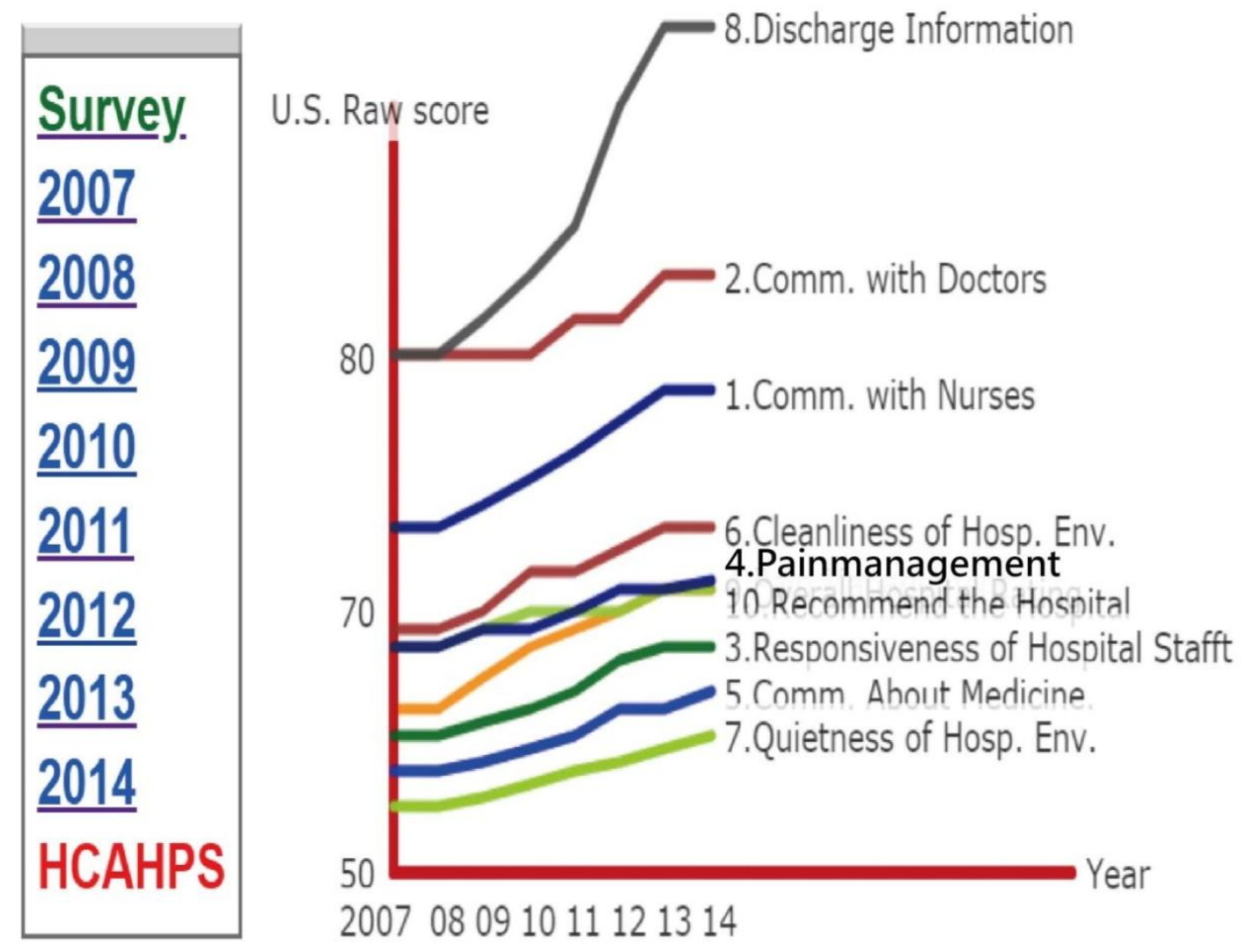

Fig-1: Trends of each domain across years

\section{Examination of item scores addible}

The data metric of $400(=8$ years $* 50$ states $) * 10$ domains were analyzed with the model-data fit (see Additional file 1). $\mathrm{R}^{2}$ was 0.93 with $\mathrm{F}=14.33$ ( $\left.\mathrm{p}<0.001\right)$ using $\mathrm{MS}$ Excel formula $=\operatorname{FDIST}(14.33,1,798)$ and the regression equation $\left(=\sum_{n=1}^{N} \sum_{j=1}^{L}\left(E_{n j}-\overline{X_{i j}}\right)^{2} / \sum_{n=1}^{N} \sum_{j=1}^{L}\left(X_{n j}-\overline{X_{i j}}\right)^{2}\right)$. The $t$-value of the two item difficulties $(-0.40$ and -0.77 logits for Communication with Nurses and Communication with Doctors) was $1.72 \quad(=$ $(-0.40-0.77) / \sqrt{1 / 48.2+1 / 36.3}), p=0.09$ using MS Excel formula $=$ TDIST $(1.72,798,2)$, not significantly different from each other using the traditional $t$-test.

A Wright map shows that the ten domains and a single construct when referring to INFIT MNSQs $<1.5$; the state distribution ranging from 0.6 to 2.0 logits is also shown (Figure 2, left). A Wright map is showing the result in 2013 presents on the Google maps at the reference [25]. 


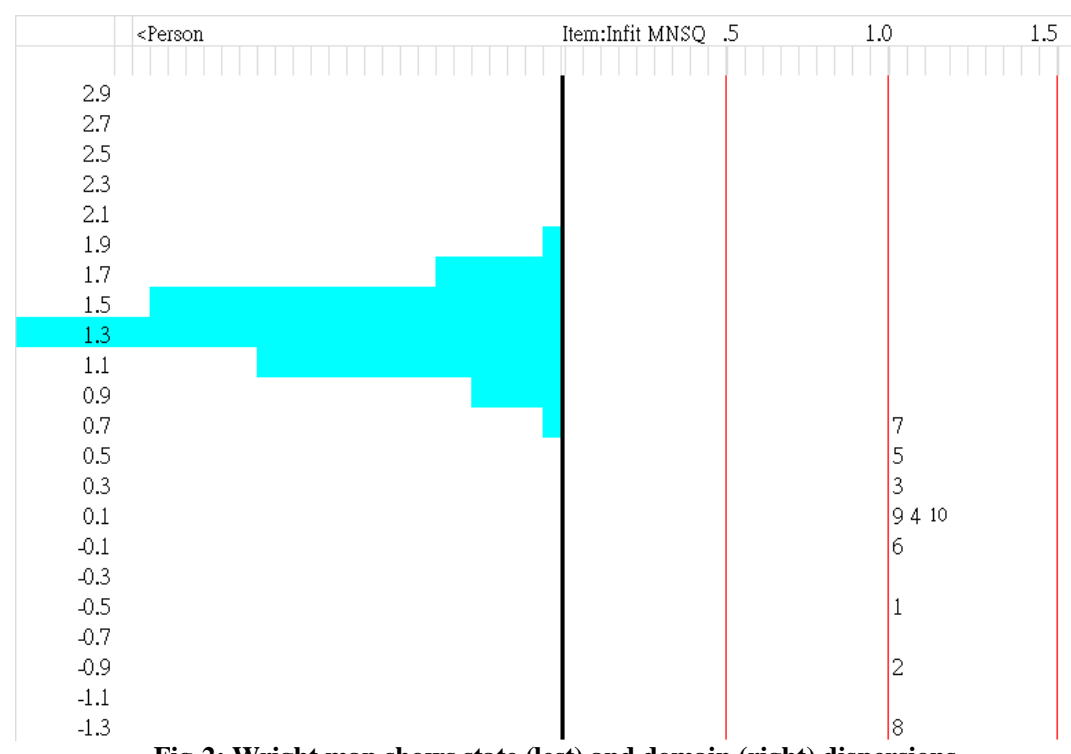

Fig-2: Wright map shows state (lest) and domain (right) dispersions

\section{Picker's item-by-item box plots}

An example was illustrated by the state of Alabama 2014; the Outfit MNSQ is 2.29 (>2.0) with an average composite score 72.9 (=1.04 logits). We can see in Figure-3 that one domain (i.e., Q7 Quietness of Hosp. Env.) show a significant high Zscore (>2.0) and present unexpected levels of inpatient experience perception in comparison to other states. It is worth noting that Outfit MNSQs (in Figure-3) [26] greater than 2.0 for a specific state indicates the responses with aberrant or careless behaviors [21], which means different response pattern of the ten domains are found in comparison with other states.

From the Figure-3 we also see that the most difficult domain (i.e., with the least score mean and a high Rasch logit calibration at 0.86 ) was Q9 (Overall hospital rating). The easiest ones (i.e., with the highest score mean) were Q8 (Discharge Information, -0.91 logits) and Q2 (Communication with Doctors, -0.56 logits).

In addition, most of those domain scores were looked like normally distributed. The other two (Q3 Responsiveness of Hospital Staff and Q9 Care Transition) were slightly negatively distributed when we see them on the Google Maps at the reference [26] if the functionalities of zoom-in and Zoon-out are manipulated.

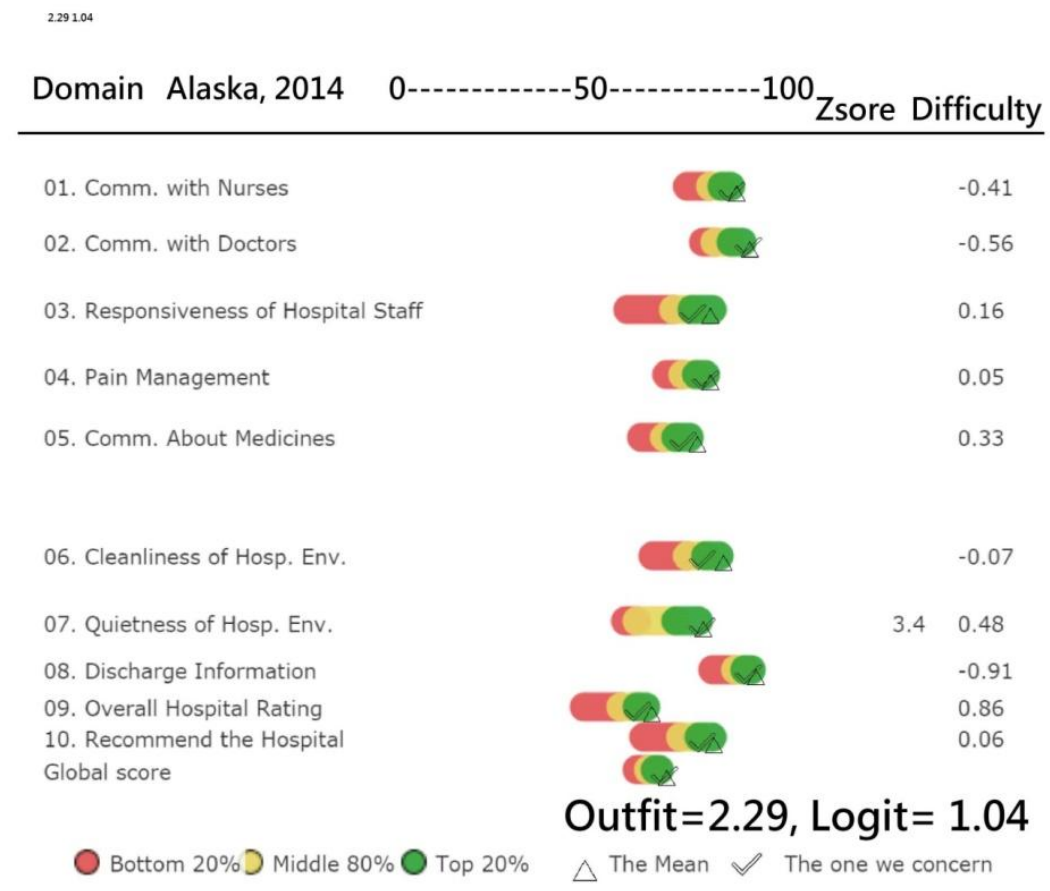

Fig-3: EPIE item-by-item box plots for Alaska 2013 with a performance at 1.03 logits and an Outfit MNSQ 2.38 


\section{A dashboard for comparing results of each US state}

An online dashboard on inpatient perceptions of hospitalization across 2007 to 2014 for US 50 states was shown in Figure-4 [27], through which we can click the icon on the year to change the feature of the US states on the dashboard. The bigger bubbles mean the better performance in the healthcare service. The triangle connected with lines indicates the bigger (or smaller) top (or bottom) three states in healthcare performance. The Wright map (Figure-2) and the KIDMAP box pot (Figure-3) can be linked via the map in Figure-4.

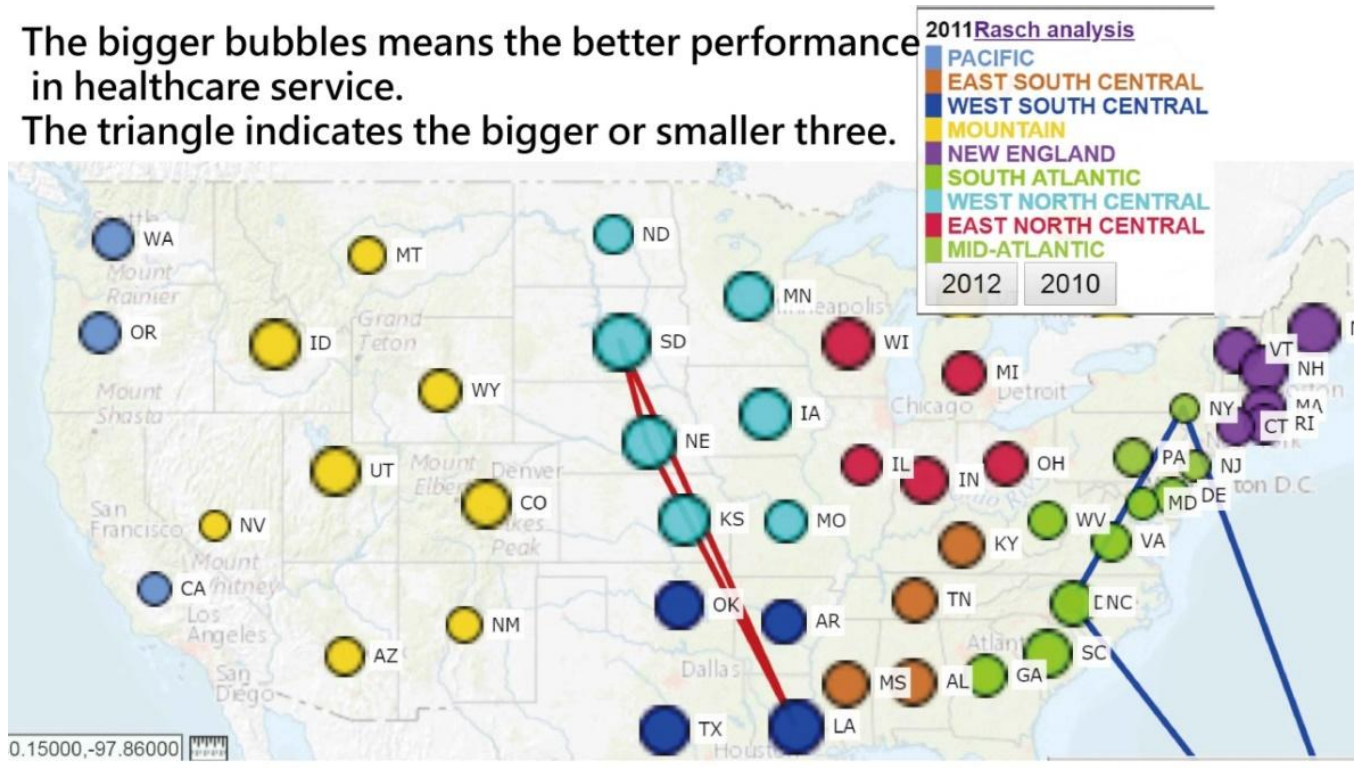

Fig-4: Dashboard on inpatient perceptions of hospitalization across 2007 to 2014 for US 50 states, clicking the icon on year to change the dashboard

\section{An online assessment using smart phones}

After scanning a QR-code shown in Figure-5, we can directly assess the inpatient hospitalization experience on the HCAHPS 25-item questionnaire by smart phones. In which, four questions will immediately redirect to the next specific questions according to the answer response. The respondents are easily confused when encountering the skip question in a paper-based survey. We demonstrated an interactive prompt module for gathering feedback about hospitalization experience from patients.

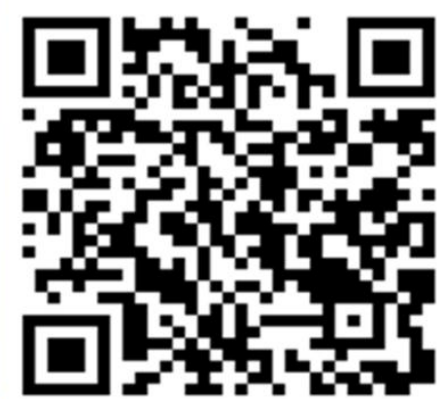

HCAHPS inpatient experience survey

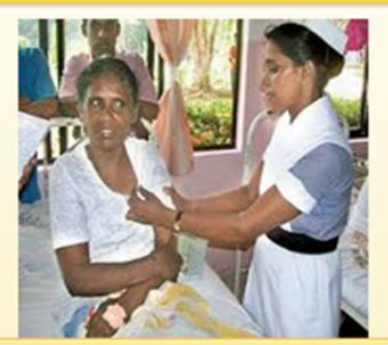

1. During this hospital stay, how often did nurses:

treat you with courtesy and respect?

Never

Sometimes

Usually

Always

Fig-5: A snapshot of an HCAHPS inpatient experience question and answers on a smartphone 


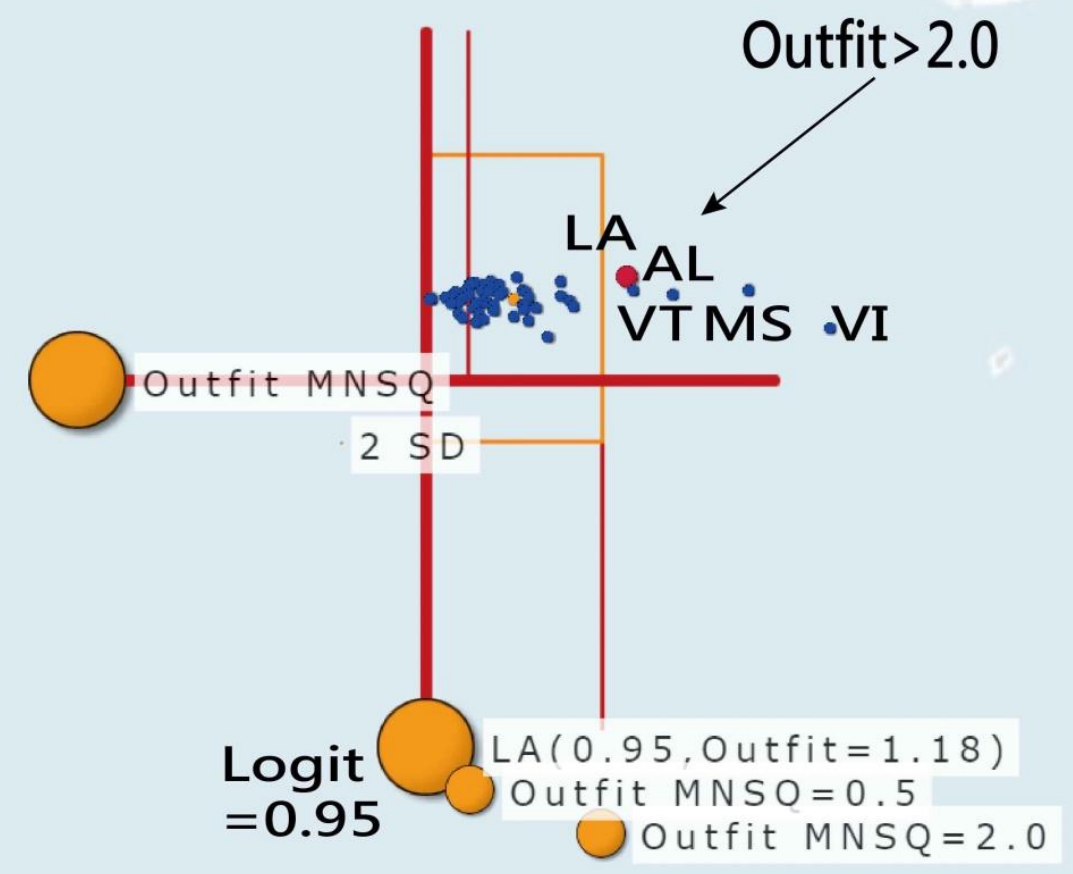

Fig-6: An Outfit map showing the aberrant pattern responded by States with the indicator of Outfit MNSQ >20.0

\section{DISCUSSION}

After analyzing the HCAHPS inpatient hospitalization experience data, we found that all domain scores but the domain nine are increasing from 2007 to 2014, and verified that the dimension scores of the 50 states were unidimensional and could be combined to interpret patient satisfactions of each state. The data also presented an online dashboard on the Google maps that can be used to compare their performance scores with the Rasch Outfit MNSQ to show the best and the worst states (Figure-3). We also demonstrated an app that can immediately gather feedback from patients using smartphones.

There are many surveys about patients' perceptions of their hospital experience. Most of them were administered by postal mail and response rates varied widely, from very low to relatively high [28]. The HCAHPS 2013 survey had an average response rate of 32\% [8]. The first public HCAHPS report was in 2007. In contrast, the UK's NHS led the way internationally in mandating a national patient survey program in 2001 [29]. The Picker Institute [7] has some survey tools that target both inpatient and outpatient experience. A growing number of people are using the Internet as a platform to describe their healthcare in the UK [30]. Similarly, in the USA, more than eight out of 10 adults regularly use the Internet [31]. However, we have not seen any performance report that can adopt advanced computer science, like Rasch model (Figure 2 and Figure-3) on the Google maps [27], to produce an interactive data visualization (Figure-4) focused on inpatient perception on hospitalization.

Furthermore, smartphones are becoming ubiquitous [32-35]. Now is the time to develop an online survey app that uses smartphones to collect patient feedback. The reason is attributable not only to studies [36-38] which report that Internet-based online ratings correlate well with the national traditional national surveys and have similar response rates but also to hospitals' need for patient feedback. The results should prove useful when patients choose healthcare providers and when clinicians want to improve the quality of their services.

In the current study, we programmed an MS Excel module, as well as an online representation on the Google, maps to deal with continuous item responses using the probabilistic modeling like Likert-type responses in a healthcare setting $[1,16]$. The average scores shown in Figure 3 are meaningful when data form a one-dimensional measurement(see Figure 3), and the states shown at the right-hand side of the scatter plot beyond 2.0 in Figure 6 on the Google maps[39] can be easily interpreted as an unusual pattern in response to domains. Linacre [22] says that Outfit mean-squares (MNSQs) greater than 2.0 deviating from Rasch model indicate aberrant responses. 
The way we incorporated Rasch analysis with Google Maps is unique in academics. Another strength and feature of this study are that Google Maps and the mp4 videos are provided and linked in references [24-27] for readers interested in manipulating the link in their ways as a dashboard. The state distribution in Figure 4 is merit in quickly understanding the feature of the state performance on patient expectations, experience, and satisfaction. One picture is worth ten thousand words. We hope following studies can report other types of information using Google API (application programming interface) to readers in the future. The study data are provided in Additional file 4.

The interpretation and generalization of the conclusions should be cautious. This study has some limitations. First, we have not mentioned that to use an online survey using smartphones, patient email and postal mail addresses should be asked for and confirmed in advance when they are admitted to the hospital and that an E-Coupon can be jointly used with a QR-code in an email when sending and receiving a solicited survey notice. Second, the HCAHPS items (see Addition all file 3) should be validated when used in different nations. For instance, item 11 (How often did you get help in getting to the bathroom or in using a bedpan as soon as you wanted?) can be revised to this like "Did you get immediate nursing help medical care when you needed it?”. We recommend using the graphical representation (Figure-2) and computer module (Figure-5) to develop an item pool used for online computerized adaptive testing in the future. Third, the data were extracted from the HCAHPS website. It is worth noting that any generalization should be made in the similar feature based on the healthcare service and the period. More studies are required to verity the quality-of-care among US states in the future.

\section{Conclusions}

A novel smartphone app designed by authors was used to acquire data from patients about their hospitalization experience. We verified that the ten domain scores reporting patient satisfaction with the performances of doctors, nurses, and hospitals in 50 US states could be combined and used to construct a unidimensional scale. It is possible to collect these data using Internet methods and smartphones and to give patients a clear idea of where to get the best care using EPIE item-by-item box plots.

\section{LiST OF ABbREVIATIONS}

EPIE: The England Picker Institute Europe

HCAHPS: Hospital Consumer Assessment of Healthcare Providers and Services

IVR: interactive voice recognition

MERS: Middle East respiratory syndrome

MNSQ: mean square

OI: outlier index

SD: standard deviation

TOC: transformed outlier coefficient

VBA: Visual Basic for Applications

\section{Declarations}

Ethics approval and consent to participate: Not applicable.

All data are publicly come from the HCAHPS website.

Consent to publish: Not applicable.

Availability of data and materials: All data used in this study is available in Additional files.

Competing interests: The authors declare that they have no competing interests.

Funding: There are no sources of funding to be declared.

\section{Authors' Contributions}

TW conceived and designed the study, SB interpreted the data, and Both of authors drafted the manuscript as well as approved the final manuscript.

ACKNOWLEDGEMENTS: We thank Frank Bill who provided medical writing services to the manuscript. 


\title{
Authors' Information
}

TW is an assistant professor at ChiMei Medical Center, Taiwan. He is an expert in computer science and Rasch modeling, mainly in the field of data analysis using the statistical technique. SB is a medical doctor with Ph.D. working as a specialist in ChiMei Medical Center, Taiwan.

\author{
Additional files \\ Additional file 1: \\ MP4. The Rasch continuous item response module \\ http://www.healthup.org.tw/marketing/course/marketing/HCAHPS2014.mp4
}

\section{Additional file 2:}

MP4. Rasch analysis on the Google maps for this study

http://www.healthup.org.tw/marketing/course/marketing/RaschHCAHPS.mp4

\section{Additional file 3:}

PDF. The US HCAHPS inpatient experience questionnaire

\section{Additional file 4:}

Xls. The study dataset

\section{REFERENCES}

1. Kaltoft, M. K., Nielsen, J. B., Salkeld, G., \& Dowie, J. (2014). Increasing User Involvement in Health Care and Health Research Simultaneously: A Proto-Protocol for" Person-as-Researcher" and Online Decision Support Tools. JMIR research protocols, 3(4), e61.

2. Marshall, M. N., Shekelle, P. G., Leatherman, S., \& Brook, R. H. (2000). The public release of performance data: what do we expect to gain? A review of the evidence. Jama, 283(14), 1866-1874.

3. Mukamel, D. B., \& Mushlin, A. I. (2001). The impact of quality report cards on choice of physicians, hospitals, and HMOs: a midcourse evaluation. The Joint Commission journal on quality improvement, 27(1), 20-27.

4. Beaulieu, N. D. (2002). Quality information and consumer health plan choices. Journal of health economics, 21(1), 43-63.

5. Fowles, J. B., Kind, E. A., Braun, B. L., \& Knutson, D. J. (2000). Consumer responses to health plan report cards in two markets. Medical Care, 469-481.

6. Schauffler, H. H., \& Mordavsky, J. K. (2001). Consumer reports in health care: do they make a difference?. Annual review of public health, 22(1), 69-89.

7. EPIE. Patient survey report - adult inpatients. Picker Institute Europe, Oxford. 2015/1/11 retrieved at http://www.pickereurope.org/

8. HCAHPS. Hospital Consumer Assessment of Healthcare Providers and Services. Current Table. December 2014 Public Report: January 2013 - December 2013 discharges. Available at http://www.hcahpsonline.org/en/summaryanalyses/.

9. Jenkinson, C., Coulter, A., Bruster, S., Richards, N., \& Chandola, T. (2002). Patients' experiences and satisfaction with health care: results of a questionnaire study of specific aspects of care. Qual Saf Health Care, 11(4), 335-339.

10. Goldstein, E., Farquhar, M., Crofton, C., Darby, C., \& Garfinkel, S. (2005). Measuring hospital care from the patients' perspective: An overview of the CAHPS ${ }^{\circledR}$ hospital survey development process. Health services research, 40(6p2), 1977-1995.

11. Chien, T. W., Wang, W. C., Wang, H. Y., \& Lin, H. J. (2009). Online assessment of patients' views on hospital performances using Rasch model's KIDMAP diagram. BMC health services research, 9(1), 135.

12. Karabatsos, G. (2003). Comparing the aberrant response detection performance of thirty-six person-fit statistics. Applied Measurement in Education, 16(4), 277-298.

13. HCAHPS. HCAHPS Hospital Survey Fact sheet: August 2013. 2015/1/11 retrieved at http://www.hcahpsonline.org/en/hcahps-star-ratings/

14. Tran, K., Morra, D., Lo, V., Quan, S. D., Abrams, H., \& Wu, R. C. (2014). Medical students and personal smartphones in the clinical environment: the impact on confidentiality of personal health information and professionalism. Journal of medical Internet research, 16(5), e132.

15. Rasch, G. (1960). Probabilistic models for some Intelligence and attainment tests. Chicago: University of Chicago Press.

16. Chien, T. W., Shao, Y., \& Kuo, S. C. (2017). Development of a Microsoft excel tool for one-parameter Rasch model of continuous items: an application to a safety attitude survey. BMC medical research methodology, $17(1), 4$.

17. Bond, T. G., \& Fox, C. M. (2001). Applying the Rasch Model: Fundamental Measurement in the Human Sciences Lawrence Erlbaum Baum Associates: Hillsdale, New Jersey.

18. Wilson, M. (2011). Some Notes on the Term: "Wright Map". Rasch Measurement Transactions, $25(3): 1331$. 
19. Chien, T. W., Chang, Y., Wen, K. S., \& Uen, Y. H. (2018). Using graphical representations to enhance the quality of care for colorectal cancer patients. European journal of cancer care, 27(1), e12591.

20. Masters, G. N. (1994). KIDMAP - a history. Rasch Measurement Transactions, 8(2):366.

21. Chien, T. W., Wang, W. C., Lin, S. B., Lin, C. Y., Guo, H. R., \& Su, S. B. (2009). KIDMAP, a web based system for gathering patients' feedback on their doctors. BMC medical research methodology, 9(1), 38.

22. Linacre, J. M. (2002). Optimizing rating scale category effectiveness. Journal of applied measurement, 3(1), 85106.

23. Dasgupta, S., Vaughan, A. S., Kramer, M. R., Sanchez, T. H., \& Sullivan, P. S. (2014). Use of a Google Map tool embedded in an internet survey instrument: is it a valid and reliable alternative to geocoded address data?. JMIR research protocols, $3(2)$, e24.

24. Chien, T. W. (2018). Trends of each domain across years on the Google maps. 2018/2/22 available at http://www.healthup.org.tw/gps/CMSlines2014.htm

25. Chien, T. W. (2018). Wright map shown on the Google maps. 2018/2/22 available at http://www.healthup.org.tw/kpiall/wrightmapb.asp?repno=3\&myear=2013

26. Chien, T. W. (2018). A KIDMAP box plot shown on the Google maps. 2018/2/22 available at http://www.healthup.org.tw/kpiall/CMSlinesbarchart.asp?repno=3\&kid=446\&myear=2013

27. Chien, T. W. (2018). A Google-map dashboard on inpatient perceptions of hospitalization across 2007 to 2014 for US $\quad 50 \quad$ states. 2018/2/22 available at http://www.healthup.org.tw/gps/healthservice.asp?journal=healthservice $\&$ myear=2011\&df=1907\&dt=2014\&zsize= $1 \& \operatorname{cen} 1=40.15 \& \operatorname{cen} 2=-97.86$

28. Debra de Silva. (2013). Measuring patient experience. The Evidence center.

29. Greaves, F., \& Millett, C. (2012). Consistently increasing numbers of online ratings of healthcare in England. Journal Med Internet Res, 14:e94.

30. Pew Research Center. (2012). Demographics of Internet users. Washington DC: Pew Research Center.

31. McBride, D. L., LeVasseur, S. A., \& Li, D. (2015). Non-work-related use of personal mobile phones by hospital registered nurses. JMIR mHealth and uHealth, 3(1), e3.

32. Tran, K., Morra, D., Lo, V., Quan, S. D., Abrams, H., \& Wu, R. C. (2014). Medical students and personal smartphones in the clinical environment: the impact on confidentiality of personal health information and professionalism. Journal of medical Internet research, 16(5), e132.

33. Greaves, F., Ramirez-Cano, D., Millett, C., Darzi, A., \& Donaldson, L. (2013). Harnessing the cloud of patient experience: using social media to detect poor quality healthcare. BMJ Qual Saf. 22(3): 251-255.

34. Njuguna, H. N., Caselton, D. L., Arunga, G. O., Emukule, G. O., Kinyanjui, DK, Kalani, R. M., Kinkade, C., Muthoka, P. M., Katz, M. A., Mott, J. A. (2014). A comparison of smartphones to paper-based questionnaires for routine influenza sentinel surveillance, Kenya, 2011¿2012. BMC Med Inform Decis Mak. 14(1):107.

35. Greaves, F., Pape, U. J., King, D., Darzi, A., Majeed, A., Wachter, R. M., \& Millett, C. (2012). Associations between Internet-based patient ratings and conventional surveys of patient experience in the English NHS: an observational study. BMJ Qual Saf, 21(7): 600-605.

36. Kamo, N., Dandapani, S. V., Miksad, R. A., Houlihan, M. J., Kaplan, I., Regan, M., ... \& Sanda, M. G. (2010). Evaluation of the SCA instrument for measuring patient satisfaction with cancer care administered via paper or via the Internet. Annals of Oncology, 22(3), 723-729.

37. Ritter, P., Lorig, K., Laurent, D., \& Matthews, K. (2004). Internet versus mailed questionnaires: a randomized comparison. Journal Med Internet Res. 6(3):e29.

38. Noel, Y., \& Dauvier, B. (2007). A Beta item response model for continuous bounded responses. Applied Psychological Measurement; 31:47

39. Chien, T. W. (2018). An Outfit maps for the State LA on the Google maps. 2018/2/22 available at http://www.healthup.org.tw/kpiall/outfitmapb.asp?repno=3\&myear=2013\&kid=LA. 